\title{
Recompensas económicas en la academia: análisis de literatura empírica sobre el pago por mérito en universidades de siete países
}

\author{
MÓNICA PATRICIA BONIFAZ CHIRINOS* \\ Pontificia Universidad Católica del Perú \\ Recibido el 17-08-2016; primera evaluación el 29-05-2017; \\ segunda evaluación el 25-07-2017; tercera evaluación el 02-11-2017; \\ aceptado el 15-11- 2017
}

\section{RESUMEN}

El estudio presenta una revisión selectiva de la literatura que expone y analiza los resultados de diez investigaciones empíricas sobre las consecuencias de la aplicación de incentivos económicos en la academia, en un contexto de transformación de la organización universitaria a nivel global. La primera parte presenta una breve descripción de los sistemas de incentivos denominados pago por méritos, sus principales características y los objetivos de su implementación. La segunda parte expone los principales hallazgos de investigaciones desarrolladas en EUA, Alemania, Australia, Estonia, Pakistán, Argentina y México, y los efectos reportados en la productividad, la motivación y la satisfacción del profesorado. Concluye con la discusión de los aspectos críticos en la implementación y evaluación de los sistemas de pago por mérito.

Palabras clave: estímulos económicos, salario de profesores, educación superior universitaria, administración universitaria, organización universitaria.

\footnotetext{
* Con Maestría en Ciencias en Gestión de la Información por la Universidad de Sheffield en Inglaterra. Diplomada en Finanzas de Centrum Católica y estudios de Especialización para Decanos en Gestión Universitaria en la Universidad de Saarbrücken en Alemania y Universidad de Alicante en España. Expresidenta de la Comisión de Gobierno de la Facultad de Gestión y Alta Dirección de la Pontificia Universidad Católica del Perú, directora de Educación Continua en la misma universidad. Contacto: mbonifaz@pucp.edu.pe.
} 


\title{
Economic rewards in academia: analysis of empirical literature on merit pay in universities in seven countries
}

\begin{abstract}
The article presents a selective review of the literature that exposes and analyzes the results of ten empirical studies on the consequences of the application of economic incentives in academia in a context of global transformation of the university. The first part presents a brief description of incentive systems known as merit pay plans, their main characteristics and the objectives of their implementation. The second part presents the main research findings developed in the USA, Germany, Australia, Estonia, Pakistan, Argentina and Mexico, and the reported effects on productivity, motivation and teacher satisfaction. Concludes with the discussion of critical aspects in the implementation and evaluation of merit-based payment systems.
\end{abstract}

Keywords: Economic incentives, teacher salary, higher education, university administration, university organization.

\section{Recompensas econômicas na academia: análise de literatura empírica sobre o pagamento de mérito nas universidades em sete países}

\section{Resumo}

O estudo descreve e analisa os resultados de um conjunto de pesquisas empíricas sobre as consequências da aplicação de incentivos económicos para os professores universitários, num contexto de reforma do ensino superior a nível mundial. A primeira parte apresenta uma breve descrição dos esquemas de incentivos chamado pagamento de mérito, suas principais características e objectivos da sua implementação. A segunda parte apresenta os principais resultados de pesquisas realizadas em os EUA, Alemanha, Austrália, Estônia, Paquistão, Argentina e México, e seus efeitos sobre a produtividade, motivação e satisfação. Conclui com a discussão de aspectos críticos na implementação e avaliação de sistemas de pagamento baseados em mérito.

Palavras chave: incentivos econômicos, salário do professores, universidade, administração da universidade, organização universitária. 


\section{INTRODUCCIÓN}

Las investigaciones relativas a los efectos que las recompensas económicas por desempeño superior tienen sobre la productividad de los trabajadores en las organizaciones empresariales son cuantiosas y variadas. La adopción de este tipo de estímulos se sustenta en los resultados de estudios empíricos que demuestran que este tipo de incentivos, apoyados en las teorías del refuerzo anticipado, mejoran los desempeños e incrementan la productividad tanto a nivel individual como organizacional. Sin embargo, recientes meta-análisis de la literatura parecen mostrar que estas mejoras impactan más en la cantidad del trabajo que en su calidad (Terpstra y Honore, 2009). Si bien las investigaciones sobre los alcances y efectos de los incentivos se apoyan, en su mayoría, en la economía de los recursos humanos, estudios recientes, desde la perspectiva de la psicología y la sociología, reportan que este tipo de estímulos extrínsecos pueden tener algunos efectos no deseados sobre la motivación intrínseca y la autodeterminación, factores también vinculados con la satisfacción, el compromiso del personal y su productividad en el largo plazo (Balán, 2012; Field, 2015; Robbins y Judge, 2013).

El objetivo de la presente revisión selectiva de la literatura es recoger y sistematizar los principales hallazgos y planteamientos de recientes, pero aún insuficientes, investigaciones de naturaleza empírica sobre las posibles consecuencias de la aplicación de recompensas económicas por desempeño superior en las organizaciones universitarias, específicamente, como componentes en los sistemas de remuneraciones académicas, bajo la modalidad denominada pago por mérito (del inglés "merit-pay»). Este tema resulta de particular interés en el contexto de reformas locales y globales que afectan a la institución universitaria (Altbach, 2012), y es especialmente relevante a las universidades peruanas en el marco del proceso de reforma de la educación superior y de la aprobación de la Nueva Ley Universitaria 30220, que establece, taxativamente, que la investigación es una función fundamental de la universidad y que, para el acceso y la progresión en la carrera profesoral, son indispensables los requisitos de profesionalización (i.e. grado doctoral) y productividad académica medida a través la publicación de investigaciones en revistas indizadas ${ }^{1}$.

\footnotetext{
La Nueva Ley Universitaria 30220, promulgada en julio de 2014, establece la obligatoriedad de la investigación en las universidades y reconoce la relevancia de la profesionalización del profesorado universitario; asimismo reconoce el derecho a una bonificación adicional hasta del $50 \%$ para los docentes investigadores.
} 


\section{El PAGO POR MÉRITO: CARACTERÍSTICAS Y ALCANCES}

El pago por mérito, del inglés «merit pay» (se usará el termino en español de ahora en adelante) es el nombre que recibe, en el entorno académico, el sistema de recompensas económicas basado en el desempeño superior de los docentes (medido de manera relativa contra el promedio de desempeńo del total del profesorado) o por productividad académico científica (medido en base a número de publicaciones que cumplen con determinados requisitos y características). Este tipo de sistemas operan como señales de la organización sobre los resultados esperados de sus miembros en función de los objetivos trazados (Türk, 2008), y responden a políticas institucionales para incrementar la productividad académico-científica, mecanismo para atraer y retener a académicos de prestigio, así como a políticas nacionales para impulsar la productividad científica mediante el acceso a fondos públicos concursables (Backes-Gellner y Schlinghoff, 2010; Galaz-Fontes y Gil-Antón, 2013; Sawar y otros, 2014).

De acuerdo con Field (2015), el pago por méritos se introduce en la academia en la década de los 90, bajo la influencia de los principios de la Nueva Gestión Pública; desde esta perspectiva, el incremento en las remuneraciones académicas debía darse en la medida que los académicos fueran más productivos o presentasen rendimientos superiores, vinculados, principalmente, a la cantidad de investigación producida.

Al respecto, Terpstra y Honoree (2009) señalan que los planes de pago por mérito, puestos en práctica en universidades norteamericanas, tuvieron como objetivo principal hacer al sistema universitario norteamericano más competitivo a nivel global. Los autores señalan que, de los casos analizados en universidades de los Estados Unidos de América, se pudo observar que los sistemas de recompensas más eficaces presentaban, al menos, las siguientes características: que el monto del pago por mérito fuera significativo con relación a la remuneración base; que la variación de la escala de incentivo en función al nivel de logro fuera, también, significativa; que los mecanismos de evaluación fueran transparentes y sencillos; que las condiciones fueran claramente enunciadas antes del proceso de evaluación, incluidas las metas y objetivos; que existiera retroalimentación luego del proceso de evaluación; que el salario base no fuera demasiado bajo y que la presencia de sindicatos fuera menor o no existieran. En resumen, que el éxito o efectividad de estos sistemas radica en su objetividad, la facilidad de comunicación y comprensión, la precisión de su medición y en disposición positiva de sus posibles receptores. 
Balán (2012) señala que, en el caso de las universidades latinoamericanas, el punto de partida de los sistemas de pago por mérito es diferente al de los países anglosajones y europeos. El investigador explica que, durante las décadas de 1970 y 1980, las remuneraciones docentes, en varios países de América Latina, sufrieron serios deterioros ante la incapacidad de los gobiernos para sostener, menos aún impulsar el desarrollo de los sistemas universitarios públicos, situación que afectó, además, al profesorado, y la cantidad y calidad de su dedicación a la docencia y la investigación.

El autor señala que, en un contexto regional caracterizado por procesos de reformas neoliberales, en países como Argentina, Brasil, México, Colombia y Chile, se implementaron reformas estructurales con la intención de recuperar y elevar la calidad de sus organizaciones universitarias y sus docentes. Ante ello, como parte de la política pública de educación universitaria, surgieron los programas de estímulo para el desarrollo de la universidad, entre los que destacan el programa desarrollado por el gobierno de México, a través del Sistema Nacional de Investigación (SNI), y el de Argentina, por su Consejo Nacional de Investigación (CNI). Ambos, en la década de 1990, incorporaron sistemas de compensación económica variable por desempeño académico en los que se incluyeron las prácticas de revisión por pares y el pago por mérito para los mejores docentes investigadores de dichos países.

Gil-Antón (2011) señala que estos pagos por mérito, en el caso mexicano, podían llegar a representar casi una remuneración adicional equivalente al ingreso base de los docentes. En el caso argentino, Sarthou (2016) señala que el sistema de pagos por mérito no solo mejoró los ingresos de los profesores, sino también la productividad y el prestigio de las universidades públicas. Por su parte, García de Fanelli (2012) al describir el sistema de compensación docente argentino, precisa que solo los académicos a tiempo completo considerados como «docentes investigadores» y registrados en el CNI percibieron ingresos adicionales a su salario base y que esta condición determinó que sus compensaciones fueran superiores al promedio de ingresos de sus colegas académicos en la misma categoría.

Bajo una lógica dominante de universidad orientada a la investigación, en la que la visibilidad y la competitividad internacional son factores relevantes, la carrera académica y sus mecanismos de compensación se están transformando, y las universidades están adoptando rápidamente nuevas políticas de remuneraciones que consideran un componente fijo (definido como básico) y uno variable de acuerdo a productividad académica (Altbach, 2012, pp. 12-13). Estos cambios, aún recientes, están siendo evaluados con relación a sus impactos en la productividad académica pero también sobre la motiva- 
ción, el compromiso y la estructura tradicional de la función académica en la universidad (Galaz-Fontes y Gil Antón, 2013; Backes-Gellner y Schlinghoff, 2010; Sarwar, Aftab, Arif y Naeem (2014); Kettunen, 2015). No obstante, la relevancia del tema y su amplia discusión, estas prácticas que han sido identificadas, en diversos países, son, aún, poco analizadas de manera sistemática y mediante estudios empíricos longitudinales que permitan tener una mayor y mejor evidencia sobre los posibles efectos netos de las mismas en el desempeño individual y organizacional (Stillwell, 2003).

Con el objetivo de contribuir con la discusión sobre este tema, a continuación, se exponen y analizan diez estudios empíricos ${ }^{2}$ seleccionados a partir de una búsqueda bibliográfica en las bases de datos Web of Science (WoS) y Ebsco EDS, mediante los términos «merit pay», «incentivos económicos» y «universidades» $\mathrm{y}$ «educación superior» (en inglés y español), con restricción en el enfoque metodológico empírico y una delimitación temporal de diez años (2007-2016).

\section{Estudios en EUA, Alemania y Australia}

En EUA, Terpstra y Honoree (2009) realizaron un estudio de correlación estadística mediante encuestas remitidas por correo electrónico a una muestra de 490 académicos a tiempo completo pertenecientes a 135 instituciones universitarias con sistemas de pago por méritos, con la finalidad de conocer su percepción sobre la efectividad de los planes de pago por mérito, tanto en las áreas de investigación, docencia como servicio.

Los principales resultados de la investigación mostraron que los planes de compensación por mérito tenían un impacto positivo en la efectividad de la docencia y en la cantidad de la producción académica, pero no se correlacionaban con los niveles de compromiso (traducido del concepto engagement) o de motivación individual por realizar las funciones de docencia, investigación o servicio con una mayor dedicación y calidad.

Si bien los hallazgos coincidieron con los resultados de investigaciones similares realizadas en organizaciones privadas (en relación con la cantidad producida) destacaron además que en el entorno académico el factor motivación intrínseca tendría una mayor correlación con la calidad y la satisfacción con el trabajo. Terpstra y Honoree (2009) reportaron además que en los sistemas de pago por mérito resultaba importante considerar la cantidad de

2 Los sesgos y limitaciones propias de la selección de los artículos y documentos revisados para el presente trabajo, son de entera responsabilidad de la autora. 
incentivo monetario y el salario base de partida, la continuidad del incentivo más allá de las variaciones presupuestales de la institución, la retroalimentación por parte de los responsables de la evaluación, y adicionalmente tomar en consideración el factor motivacional.

Leech, Haug, Iceman-Sand y Moriarty (2015) presentan los resultados de su estudio, también en EUA, sobre la relación entre el cambio en la clasificación de la universidad sujeto de estudio, de acuerdo a la clasificación Carnegie, y los cambios en la productividad académica de los docentes. En relación con la clasificación universitaria los autores detallan que en la década de 1970, la Comisión Carnegie para la Educación Superior, desarrolló un marco de clasificación para las instituciones de educación superior, que permitía diferenciar los grados obtenidos por los estudiantes; esta clasificación incluía diferentes categorías desde colleges de formación hasta universidades de investigación.

El caso analizado es una universidad norteamericana que decide pasar de la clasificación de universidad de doctorado-investigación, a universidad de investigación (el siguiente y más alto nivel). Esta decisión implicó la introducción de un nuevo sistema de evaluación de desempeño, en el que comités de pares ad-hoc evaluaban los resultados de productividad y los pagos por méritos adicionales a la remuneración base. Leech et al. (2015) analizaron si efectivamente se produjo un incremento en los niveles productividad académica en los años posteriores a la aplicación de los incentivos y al cambio de clasificación a universidad de investigación. Para ello los autores realizaron un análisis correlacional entre el sistema de pago por mérito y la producción académica de los docentes pertenecientes a la Escuela de Educación durante un periodo de diez ańos, con datos sobre la cantidad y el tipo de publicaciones reportadas en los CV de los profesores.

Los resultados del análisis no mostraron variaciones significativas ni en la cantidad ni en la combinación del tipo de publicaciones. Si bien el hallazgo resultaba interesante en comparación a otros estudios similares, este no podía ser considerado representativo pues los mismos autores reportaron limitaciones en el sistema de captación de datos a partir de los curriculum vitae, a que el estudio no había incorporado la variable de significancia de la recompensa económica en términos monetarios, ni el histórico de productividad, que en promedio era bajo en comparación con las productividades de otras escuelas en la misma universidad, como por ejemplo la Escuela de Medicina.

Backes-Gellner y Schlinghoff (2010) realizaron un estudio empírico comparado sobre los posibles efectos de las recompensas económicas por producción académico-científica en el comportamiento de 189 académicos de universidades alemanas y 112 pertenecientes a universidades norteamericanas, 
en ambos casos de las carreras de economía y negocios, y en proceso de acceder al tenure track (en adelante carrera ordinaria).

El estudio se basa en el seguimiento de carrera reportado por los profesores en su CV, su producción académica y en la aplicación de una encuesta por correo electrónico. Tomando como antecedente la Teoría del Torneo de Hartford, el objetivo de esta investigación fue observar el comportamiento de la productividad académica ante los incentivos económicos y el progreso en la carrera académica, vistos como procesos de competencia interna y externa. Los resultados del estudio mostraron que la productividad académica de los profesores de universidades norteamericanas se correlacionaba positivamente con el sistema de recompensas monetarias y con los ascensos profesorales en el corto plazo, pero que una vez logrado el tenure su productividad tendía a estancarse o decrecer. A diferencia de los profesores de universidades alemanas con un sistema carrera progresivo y con estímulos económicos de menor magnitud, cuyas productividades no decrecían una vez logrado el tenure.

A partir de sus resultados, Backes-Gellner y Schlinghoff (2010) estimaron que dado que la mayoría de las universidades de países industrializados se inclinaban por imitar el sistema norteamericano, los académicos tenderían a adecuar sus comportamientos en función de los incentivos económicos de corto plazo, por lo que se priorizaría por publicar en desmedro de otras funciones académicas, pronosticando que la formación y otros servicios sociales provistos por la universidad serían desplazados por la política de «publicar o morir» (del inglés publish or perish).

Wilkesmann y Schmid (2012), también en Alemania, desarrollaron un estudio mediante encuestas a profesores universitarios con el objetivo de evaluar la percepción docente de la nueva política de estado por incentivar económicamente (a través de merit pay y teaching awards) la dedicación de los académicos a la función de docencia, afectada por la competencia y los incentivos por publicar. Esta investigación tuvo como población de estudio a 21000 profesores activos en las universidades alemanas, de los que se seleccionaron 8000; a estos se les remitió por correo una encuesta de medición de percepción en relación con la motivación intrínseca y extrínseca frente a la docencia, los métodos de enseńanza y los factores culturales y sociales asociadas a la misma.

El estudio obtuvo una tasa de respuesta de 13\%. Bajo el marco teórico de Principal-Agente y de la influencia de la Nueva Gestión Pública en la reforma alemana, plantearon tres grupos de hipótesis; el primero relacionado a los supuestos de la Teoría de Principal-Agente, el segundo en relación con factores motivacionales intrínsecos y el tercero en relación con factores socioculturales. En las pruebas estadísticas de regresión múltiple, los investigadores 
no hallaron influencias significativas de los incentivos económicos, tampoco de los factores motivacionales intrínsecos en su compromiso y esfuerzo por la docencia. Sin embargo, sí confirmaron sus hipótesis sobre los efectos de la estructura social y la cultura organizacional sobre el compromiso docente, concluyendo que para mejorar la docencia los estímulos más efectivos son aquellos vinculados a factores culturales, de estructura y de entorno, más no los monetarios.

Desde otra perspectiva de investigación, Field (2015) señala que los estudios de medición cuantitativa sobre la efectividad de las recompensas y la opinión de los administradores que las implementan resultan insuficientes, por lo que se requiere incorporar la opinión de los académicos para comprender, de manera integral, los efectos y consecuencias deseadas y no deseadas de los sistemas de pago por desempeño superior en la academia.

El estudio de Field (2015) realizado en una universidad pública australiana de tamaño medio, recoge la opinión de una muestra de académicos, que han recibido y no han recibido recompensas por rendimiento superior, y que pertenecen a diferentes escuelas. Para ello desarrolla una metodología cualitativa, en base a 40 entrevistas a académicos ( 20 hombres y 20 mujeres) seleccionados al azar entre diferentes escuelas, categorías y edad. Las entrevistas fueron semiestructuradas, transcritas y analizadas con el software NVivo. El estudio cualitativo diferenció dos categorías de análisis, los profesores de la Escuela de Negocios (EN) y los profesores de otras escuelas (OE).

Con relación al análisis de los académicos de la EN se observó una disposición positiva al pago por desempeńo superior, como mecanismos para atraer, motivar y retener académicos de alto nivel, mientras que los académicos de OE mostraron una fuerte oposición al pago por desempeño superior. Estos últimos, por principio, la consideraron ofensiva a la capacidad de investigación, así como inequitativa.

El estudio también recogió coincidencias entre ambos grupos, específicamente en relación con las observaciones sobre la forma de implementación del sistema; ambos grupos señalaron que los sistemas de pago por mérito eran poco transparentes, complejos y subjetivos, además que privilegiaban la cantidad sobre la calidad y adolecían de retroalimentación. Desde el punto de vista cualitativo el estudio de Field (2015) aporta con observaciones más sobre la forma que sobre el fondo del sistema, aunque como seńalan la autora, dado el tamaño de la muestra este no pretendía ser representativo de la opinión de los profesores de la universidad. 


\section{Estudios en Estonia y Pakistán}

El objetivo de la investigación de Türk (2008) fue describir y analizar los efectos del sistema de pago por desempeño implementado en la Universidad Pública de Tartu, la más antigua de Estonia. El estudio aplicó una metodología mixta de análisis documental, cuestionarios a 125 profesores de la Escuela de Economía y Negocios (ECN) y la Escuela de Educación (con una tasa de respuesta 52\%) y cinco entrevistas a responsables administrativos y académicos senior. Los resultados mostraron la aceptación general del sistema de pago por desempeńo y el reconocimiento de un impacto positivo en la motivación, así como en el incremento de la productividad académica. A pesar de los resultados favorables reportados, el estudio recogió la percepción de los profesores en relación con la complejidad del sistema, su puntuación y con la necesidad de simplificar la metodología para hacerla más confiable. La principal limitación del estudio Türk (2008) fue el tamańo de la muestra, poco representativa debido a la baja tasa de respuesta obtenida.

En Pakistán, Sarwar et al. (2014) realizaron un estudio empírico transversal a una muestra de 400 académicos de universidades públicas y privadas, con el objetivo de comparar resultados en relación con la disposición del docente a los sistemas de pago por mérito o por desempeńo. Los investigadores aplicaron cuestionarios a diferentes grupos de profesores universitarios (por grado, edad, género y afiliación a universidad pública o privada) con el propósito de recoger información sobre sus niveles de disposición, motivación y desempeño en relación con las recompensas económicas ofrecidas por sus universidades.

Este fue un estudio de alcance comparativo, como el conducido por Backes-Gellner y Schlinghoff (2010), aunque en este caso entre académicos de un mismo país, pero pertenecientes a universidades públicas y privadas. Los niveles de disposición, satisfacción, motivación y desempeño, fueron definidos como variables diferentes entre sí. Los investigadores aplicaros diferentes pruebas estadísticas como Mann Whitney U Test, Kruskal Wallis Test y Chi cuadrado. Sobre la base de 16 grupos de hipótesis, los investigadores no encontraron soporte estadístico significativo con relación al pago por mérito y la satisfacción de los docentes. Sí encontraron evidencia estadística con relación a una mejor aceptación de los incentivos económicos y su efecto en el desempeño entre los profesores de universidades públicas, así como un mayor grado de motivación en estos frente a sus pares de universidades privadas. Coincidente con los estudios previos señalados, los pagos por mérito sí mostraron correlación con el desempeño, aunque no necesariamente con la motivación y la satisfacción del personal académico. 
El estudio arrojó que los resultados de satisfacción y desempeño fueron superiores para el grupo de académicos con grado de doctor y dedicación a tiempo completo, siendo este el grupo que se mostró con mayor disposición a la aplicación de este tipo de sistema de compensaciones. Adicionalmente, la investigación comparada halló que los docentes de las universidades públicas se mostraron significativamente más dispuestos a los incentivos económicos que sus pares de las universidades privadas.

\section{ESTUDIOS REPORTADOS POR PAÍSES LATINOAMERICANOS: Argentina y MÉXICO}

Entre los países latinoamericanos de habla hispana, es de conocimiento que el sistema universitario mexicano, argentino, chileno y colombiano han incorporado los planes de incentivos por productividad académica como parte de sus políticas públicas para estimular la investigación e incrementar la producción académica-científica, y con ello impulsar la visibilidad y la reputación internacional de sus universidades (Altbach, 2012). Sin embargo, a la fecha, aún son muy pocos los estudios de carácter empírico publicados por investigadores de los países antes citados. En algunos casos, como el chileno, el argentino y el colombiano (Berrios, 2008; García de Fannelli, 2012; Pacheco, 2012) los resultados se presentan a nivel de carrera académica, por disciplina o género, pero no de manera desagregada a nivel de sistema de compensaciones fijovariable y de incentivos por productividad académica.

En Argentina, el estudio de Sarthou (2016) revisa las consecuencias del Programa de Incentivos Económicos implementado por el Ministerio de Educación de la República Argentina en la década de 1990, cuyo objetivo fue incrementar la productividad académica y el prestigio de los profesores de las universidades públicas. De acuerdo a la autora, el sistema de pago por desempeño fue introducido tanto en México como en Argentina en la década de 1990 como un instrumento de política bajo la influencia de los principios de la Nueva Gestión Pública y el desarrollo de políticas liberales en la región latinoamericana.

El Programa de Incentivos debía garantizar que, a pesar de las restricciones del presupuesto público para el sector, se incrementara la capacidad de producir conocimientos desde las universidades públicas. Si bien la literatura argentina es crítica en relación con los efectos no deseados de este programa, por estimular el trabajo individualista y por el control externo sobre el desempeńo de los profesores, Sarthou (2016) observa que, a pesar de las críticas, el programa ha prevalecido. La autora analiza, mediante estadística descriptiva, los efectos 
del programa sobre los ingresos anualizados de los docentes y los incrementos de la productividad académica, medida en número de publicaciones.

El estudio reportó que, durante el periodo de análisis, sí hubo incremento en los ingresos de los profesores investigadores, a pesar que estos variaron de acuerdo a los presupuestos asignados por los diferentes gobiernos bajo el periodo de análisis. Concluye, además, que, dado que el programa no solo ha mejorado los ingresos de los profesores, sino también la productividad y el prestigio de las universidades públicas argentinas, el sistema se mantiene con la aceptación del profesorado y con moderadas modificaciones hasta el cierre de su estudio.

En México, se han reportado los resultados de las investigaciones sobre la Universidad Autónoma Metropolitana (Gil-Antón, 2011), y el análisis del Censo Académico 2007-2008 (Galaz-Fontes y Gil-Antón, 2013); en ambos estudios el objetivo fue examinar el alcance y posibles efectos de los planes de pagos por mérito implementados en la década de los 1990.

El estudio de Gil-Antón (2011) examina el caso de la Universidad Autónoma Metropolitana (UAM) que adoptó el sistema de incentivos económicos desde 1989 con dos objetivos, el primero, mejorar los ingresos medios de sus académicos que se había visto mermado por las crisis económicas, y el segundo transformar el ejercicio de la carrera docente en una ocupación a tiempo completo orientada a resultados y con evaluación por desempeño. Este estudio de caso permitió analizar la composición de los salarios docentes y estimar que, para el nivel más alto de profesorado en México, la compensación total promedio estaba compuesta de $54 \%$ remuneración base y $46 \%$ compensación variable. Si bien este sistema ha permitido nivelar el ingreso promedio a uno de mayor poder adquisitivo, el autor seńala que la estructura fijo-variable no se garantiza un fondo pensionable atractivo para el retiro, lo que en el largo plazo podría dificultar el proceso de renovación académica.

El sistema de pago por méritos produjo, además, un cambio en la estructura de carrera profesoral, más orientada a la investigación internacional y con posiciones diferenciadas entre la docencia y la investigación, así como de diferenciación en la compensación entre disciplinas académicas, donde aquellas más profesionalizadas (como leyes, negocios o ingenierías) reportaron ingresos medios inferiores a aquellas más orientadas a la producción científica.

Adicionalmente, según Gil-Antón (2011) el sistema tiende a premiar la productividad individual (basada en elaboración de papers y asistencia a conferencias) en detrimento del trabajo académico colaborativo. Entre los principales hallazgos del autor, destaca en primer lugar que el sistema de incentivos sí ha logrado incrementar la productividad académica del país, 
además de estar respaldada por los mecanismos de evaluación por pares del SNI, y que el sistema sí ha posibilitado la mejora de la calidad académica de los productos de la investigación. En segundo lugar, señala que los académicos tienden cada vez más a concentrarse en investigaciones de corto aliento, a fin de acceder a los beneficios económicos en el corto plazo; señala que son pocos los académicos involucrados en investigaciones de largo aliento y alcance. Y tercero, con el objetivo de investigar y publicar, los docentes tienden a estar menos disponibles para la atención de estudiantes y prefieren trabajar desde sus casas, reduciendo su participación en el gobierno y la vida universitaria.

El segundo estudio, conducido por Galaz-Fontes y Gil-Antón (2013) es de mayor alcance; utiliza los datos del Censo de Académicos 2008-2009 con el objetivo de evaluar el impacto y las perspectivas de diversos sistemas de pago por méritos dirigidos a los académicos de universidades mexicanas, en el marco de los programas públicos de financiamiento por desempeño, con la participación de la SNI de México. Los autores parten del siguiente marco de referencia: primero, los planes de pago por mérito no son sostenibles a largo plazo y tienden a tener efectos sobre el desempeño individual (Kohn, 1994, citado por Galaz-Fontes y Gil-Antón, 2013); segundo, los estudios previos arrojan resultados disímiles dependiendo de las estructuras salariales diferenciadas entre diferentes países; por ejemplo, en México el incentivo económico es muy alto, casi el $50 \%$ adicional, mientras que en Canadá o en Estados Unidos de Norteamérica es menos del $10 \%$. Tercero, las políticas públicas tienden a privilegiar la investigación en los campos relacionados a las ciencias y la tecnología, en detrimento de la investigación en otras disciplinas como las ciencias sociales; y por último, los sistemas de pago por mérito no deben ser vistos de manera aislada, pues la trayectoria de la carrera académica, también constituye un mecanismo de incentivo económico (Altbach, 1991 citado por Galaz-Fontes y Gil-Antón, 2013).

El censo sobre el que Galaz-Fontes y Gil-Antón (2013) trabajan, recoge datos de más de 1770 académicos a tiempo completo en todo tipo de universidades mexicanas; muchos con formación doctoral y alta productividad académica en publicaciones de impacto. El análisis del censo, arroja los siguientes resultados: desde la entrada en vigencia del programa de pago por mérito, considerando el año base 1992 , la presencia de doctores se ha incrementado de $12 \%$ a $34 \%$; el ingreso adicional por pago por mérito es de $14 \%$ para docentes con maestría no registrados en el SIN, de $20 \%$ para los doctores no registrados en el SIN y del 50\% para los doctores registrados en el SNI. De una media de 22 horas de enseñanza y 10 horas de investigación por tiempo completo, un docente doctorado afiliado al SIN, dedica a la docencia 15 y a la investigación 20 horas. 
Del análisis de los datos censales, los autores concluyen que los programas de pago por mérito operan como un sistema de trasferencia de efectivo condicionada que privilegia el desempeño individual más que institucional; que el sistema aleja a los mejores investigadores de la docencia y desincentiva la participación de los mismos en el gobierno de su universidad, y finalmente, que el sistema ha creado las condiciones para que los académicos no encuentren mejor alternativa de retribución y compensación que la creada por los programa basados en incentivos económicos por productividad académica.

\section{DISCUSIÓN Y CONCLUSIONES}

Los procesos de reforma del sistema universitario de los últimos treinta años, tanto a nivel global como regional, han traído consigo nuevos modelos de dirección y desarrollo de la carrera académica, y transformaciones en los sistemas tradicionales de remuneraciones docentes, enfocándose cada vez más en la medición de la productividad académica, con el objetivo de impulsar el prestigio y la competitividad de las universidades (Altbach, 2012; Kettunen, 2015; Stillwell, 2003). Desde una perspectiva regional, los sistemas de compensación fijo-variable que incorporan planes de pago por mérito, dados por iniciativa del Estado con fondos públicos o con fondos de las propias universidades, han permitido compensar la pérdida del poder adquisitivo de los docentes latinoamericanos, revalorar la carrera académica y alinear los intereses institucionales con los individuales, estén estos vinculados a la función de docencia o de investigación (Galaz-Fontes y Gil-Antón, 2013; Sarthou, 2016).

No obstante, la amplia literatura sobre los cambios de los sistemas tradicionales de compensación por sistemas compuestos fijo-variable basados en desempeño superior, o por mérito, son pocas investigaciones de carácter empírico que reportan resultados sobre los efectos que estos tienen en la productividad de largo plazo, la calidad de los resultados, la satisfacción y el compromiso a nivel individual. (Backes-Gellner y Schlinghoff, 2010; Wilkesmann y Schmid, 2012; Sarwar et al., 2014)

Si bien la evidencia existente muestra la influencia positiva de los sistemas de pagos por mérito en la productividad académica de corto plazo, así como sobre la aceleración de la tasa de profesionalización (i.e. obtención de grado de doctor) y del prestigio de las universidades, no es del todo claro en qué medida las consecuencias no deseadas, como el trabajo individual, el alejamiento de la docencia y de la vida universitaria, están vinculadas más a la forma de implementación de dichos programas que a la decisión institucional de implementarlos. Por otro lado, a pesar que la literatura es crítica sobre el 
fondo y la forma de implementación de estos sistemas, el incremento de los salarios medios del profesorado los justifica como mecanismo de mejora remunerativa y los hace sostenibles en el largo plazo. (Galaz-Fontes y Gil-Antón, 2013; Sarthou, 2016; Terpstra y Honoree, 2009; Sarwar et al., 2014).

Por otra parte, resulta pertinente señalar que el rechazo de la academia hacia este sistema parece estar correlacionado con variables como el área disciplinar, la edad, el grado de formación y el tipo de institución universitaria, variables que ameritan una observación más exhaustiva, sobre todo en los procesos de diseńo o reforma de los sistemas de compensaciones. Las posiciones críticas están vinculadas, además, al posible uso de los sistemas de recompensas como mecanismo de control sobre la academia bajo una lógica de comoditización de la labor docente y de la industrialización de las funciones de docencia e investigación en las universidades (Stillwell, 2003; Galaz-Fontes y Gil-Antón, 2013, Kettunen, 2015; Sarthou, 2016).

A pesar de los hallazgos de las aún pocas investigaciones en nuestra región, existen preocupaciones razonables en términos de los efectos que estos planes tienen sobre las estructuras profesionales en las universidades, en las que se meritúa la función de investigación sobre la función docente (i.e. la investigación es económicamente mejor valorada que la docencia) o se estimula silenciosamente el alejamiento de los mejores investigadores de la cátedra y del servicio en la universidad (Backes-Gellner y Schlinghoff, 2010; Galaz-Fontes y Gil-Antón, 2013), aspectos que merecen ser analizados de manera particular en futuros estudios.

Finalmente, la ausencia de investigaciones empíricas con relación a los sistemas remunerativos y de recompensas docentes en los países latinoamericanos representa un reto y un compromiso de los investigadores de la región con el adecuado análisis y diseño de estrategias de desarrollo de la carrera académica y de gobierno universitario, en un contexto en el que los sistemas de educación superior universitaria en la región plantean nuevos escenarios para la institución universitaria, sus miembros y la sociedad. 


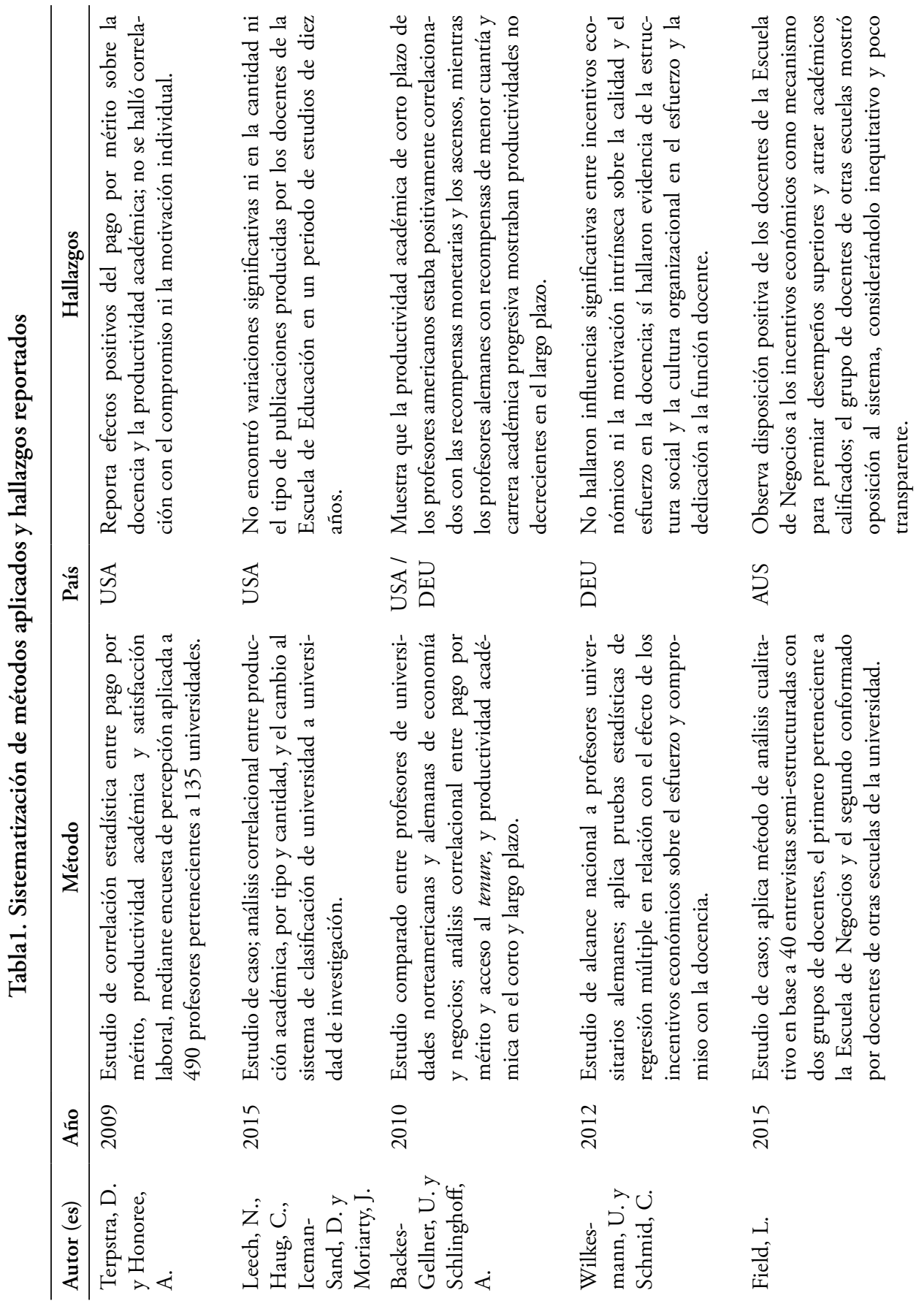




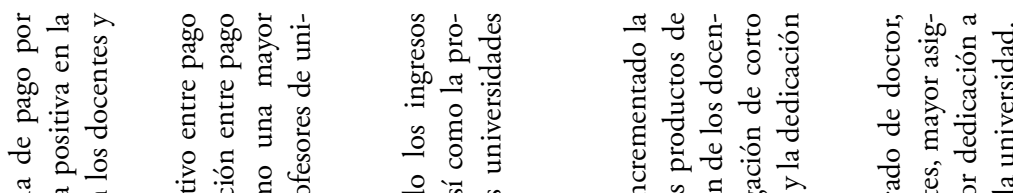

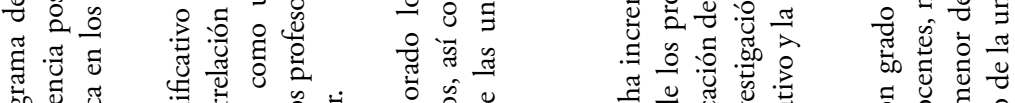

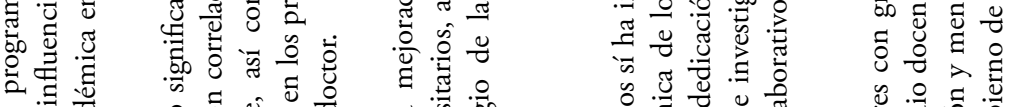

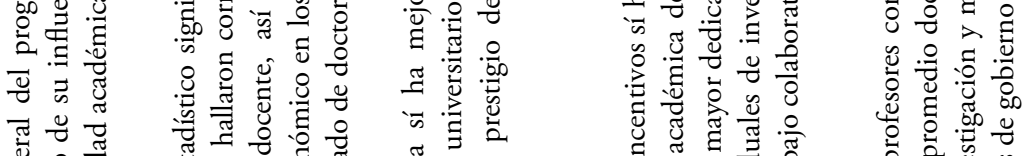

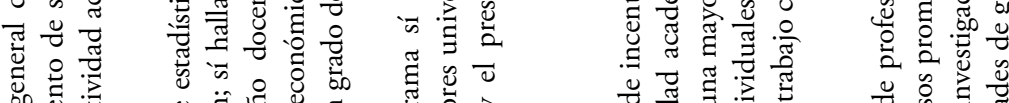

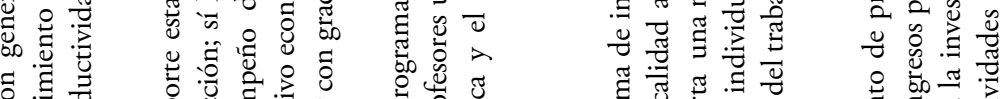

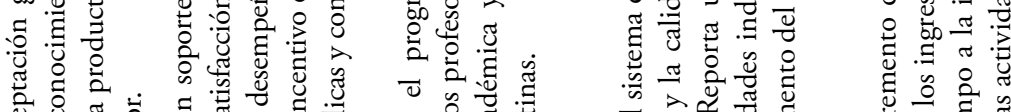

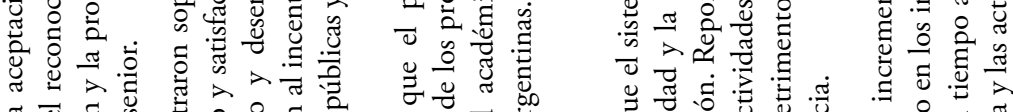

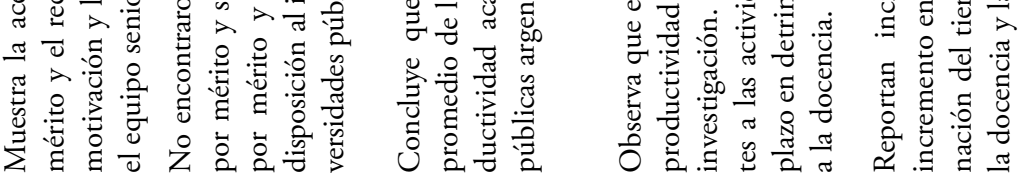

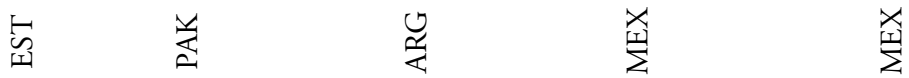

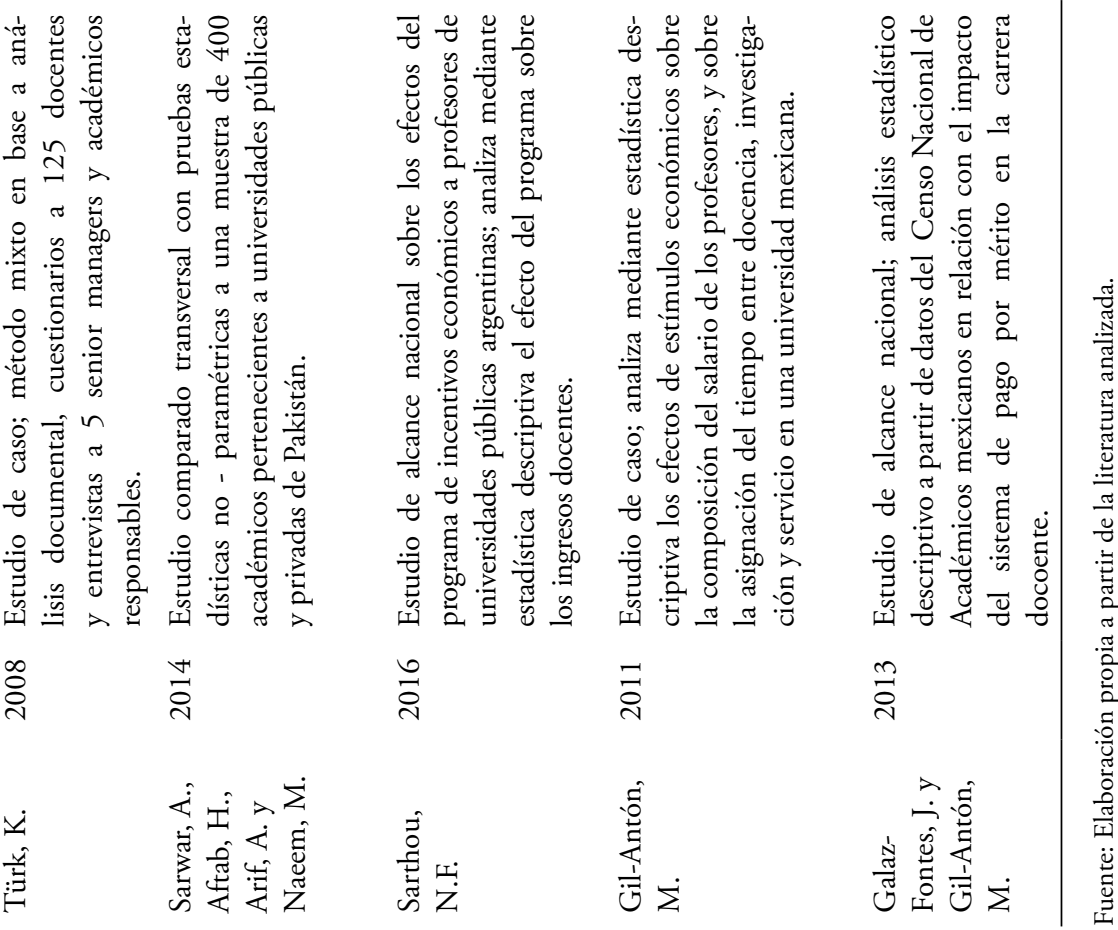




\section{REFERENCIAS BIBLIOGRÁFICAS}

Altbach, P. (ed.). (2012). Paying the professoriate: a global comparison of compensation and contracts. Nueva York: Routledge

Backes-Gellner, U. y Schlinghoff, A. (2010). Career incentives and »Publish or Perish» in German and U.S. universities. European Education, 42(3), 26-52. https://doi.org/10.2753/EUE1056-4934420302

Balán, J. (2012). Research universities in Latin America: the challenges of growth and institutional diversity. Social Research, 79(3), 741-770.

Berrios, P. (2008). The academic career: an empirical analysis on its structure and organization in Chile. Calidad en la Educación, 29, 35-62.

Field, L. (2015). Using outperformance pay to motivate academics: insiders' accounts of promises and problems. Australian Universities' Review, 57(2), 5-16.

Galaz-Fontes, J. y Gil-Antón, M. (2013). The impact of merit-pay systems on the work and attitudes of Mexican academics. Higher Education, 66, 357-374. https://doi.org/10.1007/s10734-013-9610-3

García de Fanelli, A. (2012). Labour contracts and economic incentives for Argentine university faculty. En G. Altbach (ed.), Paying the professoriate: a global comparison of compensation and contracts (pp. 37-48). New York: Routledge.

Gil-Antón, M. (2011). The merit pay system in a Mexican university: the case of Metropolitan Autonomous University. The Journal of the Professoriate, $4(2), 122-140$.

Kettunen, J. (2015). Towards the high profile of higher education institutions. Educational Alternatives, 13, 86-95

Leech, N., Haug, C., Iceman-Sand, D. y Moriarty, J. (2015). Change in classification level and the effects on research producitivity and merit scores for faculty in a school of education. Studies in Higher Education, 40(6), 10301045. https://doi.org/10.1080/03075079.2014.881341

Ley Universitaria 30220 (2014). Diario Oficial El Peruano. Lima, Perú. 9 de julio. Pacheco, I. (2012). Academic salaries in Colombia. En G. Altbach (ed.), Paying the professoriate: a global comparison of compensation and contracts (pp. 104-113). Nueva York: Routledge.

Robbins, S. y Judge, T. (2013). Comportamiento organizacional. 15 ed. México D.F.: Pearson.

Sarthou, N.F. (2016). Twenty years of Merit-Pay programme in Argentinean Universities: tracking policy change through instrument analysis. 
Higher Education Policy, 2016(29), 379-397. https://doi.org/10.1057/ s41307-016-0001-0

Sarwar, A., Aftab, H., Arif, A. y Naeem, M. (2014). Performance related pay: a comparative study on public and private universities. Science International (Lahore), 26(1), 489-497.

Stillwell, F. (2003). Higher Education, Commercial Criteria and Economic Incentives. Journal of Higher Education Policy and Management, 25(1), 51-61. https://doi.org/10.1080/13600800305741

Terpstra, D. y Honore, A. (2009). Merit pay plans in Higher Education Institutions: characteristics and effects. Public Personel Management, 38(4), 55-77. https://doi.org/10.1177/009102600903800404

Türk, K. (2008). Performance appraisal and the compensation of academic staff in the University of Tartu. Baltic Journal of Management, 3(1), 40-54. https:// doi.org/10.1108/17465260810844257

Wilkesmann, U. y Schmid, C. (2012). The impacts of new governance on teaching at German universities. Findings from a national survey. Higher Education, 63, 33-52 https://doi.org/10.1007/s10734-011-9423-1 